\title{
Can Coupling Multiple Complementary Methods Improve the Spectroscopic Based Diagnosis of Gastrointestinal Illnesses? A Proof of Principle Ex Vivo Study Using Celiac Disease as the Model Illness
}

\author{
Sara J. Fraser-Miller, ${ }^{\dagger *}$ Jeremy S. Rooney, ${ }^{\dagger}$ Michael Lau, ${ }^{\ddagger}$ Keith C. Gordon, ${ }^{\dagger}$ and Michael Schultz. ${ }^{\S, l, \perp}$ \\ $\dagger$ Dodd-Walls Centre for Photonic and Quantum Technologies, Department of Chemistry, University of Otago, Dunedin, 9054, New \\ Zealand. \\ † Southern Community Laboratories, Dunedin, 9016, New Zealand. \\ $\S$ Gastroenterology Research Unit, Department of Medicine, Dunedin School of Medicine, University of Otago, Dunedin, 9054, New \\ Zealand.
}

\| Mercy Hospital, Dunedin, 9010, New Zealand.

$\perp$ Gastroenterology Department, Southern District Health Board, 9016, Dunedin, New Zealand.

\section{Contents}

Table S 1 Summary of the PLSR model performance when correlating anti-tTG antibody levels to the spectroscopic data.

Table S 2 Recalculated diagnosis performance based on the 4 level histology SVM and grouping different severities together. TP=true positive, TN = true negative, $\mathrm{FP}=$ false positive and $\mathrm{FN}=$ false negative

Table S 3 Diagnosis based SVM performances by participant (classification per participant is the majority from individual biopsies). TP=true positive, $\mathrm{TN}=$ true negative, $\mathrm{FP}=$ false positive and $\mathrm{FN}=$ false negative.

Figure S 1 PCA of the LFR spectroscopic dataset. (a) Scores plot of PC1 versus PC2 and (b) the associated loadings and comparative average spectrum per sample group. Please note the $*$ indicates rotational spectra of air.

Figure S 2 PCA of the 532R spectroscopic dataset. (a) Scores plot of PC1 versus PC2 and (b) the associated loadings and comparative average spectrum per sample group.

Figure S 3 PCA of the 532B spectroscopic dataset. (a) Scores plot of PC3 versus PC5 and (b) the associated loadings and comparative average spectrum per sample group.

Figure S 4 PCA of the NIR spectroscopic dataset. (a) Scores plot of PC1 versus PC2 and (b) the associated loadings and comparative average spectrum per sample group.

Figure S 5 Summary of the SVM-histology performance statistics versus number of techniques coupled.

Figure S 6 Example confusion matrix for the histology SVM and grouping different severities together. $\mathrm{TP}=$ true positive, $\mathrm{TN}=$ true negative, $\mathrm{FP}=$ false positive and $\mathrm{FN}=$ false negative.

Figure S 7 Summary of the SVM-diagnosis performance statistics versus number of techniques coupled....

Figure S 8 ROC area under the curve for each class from SVM classification models with 4 histological levels - individual techniques.

Figure S 9 ROC area under the curve for each class from SVM classification models with 4 histological levels - combining two techniques. ...... S-11

Figure S 10 ROC area under the curve for each class from SVM classification models with 4 histological levels - combining three techniques. .. S-13

Figure S 11 ROC area under the curve for each class from SVM classification models with 4 histological levels - combining four techniques..... S-14

Figure S 12 ROC area under the curve for each class from SVM classification models with 4 histological levels - combining five techniques..... S-15

Figure S 13 ROC curves for the individual techniques. (a) LFR, (b) FTR, (c) 532R, (d) 532B, and (e) NIR

Figure S 14 ROC curves for the paired techniques. (a) LFR FTR, (b) LFR 532R, (c) LFR 532B, (d) LFR NIR, (e) FTR 532R, (f) FTR 532B, (g) FTR NIR, (h) 532R 532B, (i) 532R NIR and (j) 532B NIR.

Figure S 15 ROC curves for the triple technique combinations. (a) LFR FTR 532R, (b) LFR FTR 532B, (c) LFR FTR NIR, (d) LFR 532R 532B, (e) LFR 532R NIR, (f) LFR 532B NIR, (g) FTR 532R 532B, (h) FTR 532R NIR, (i) FTR 532B NIR, and (j) 532R 532B NIR.

Figure S 16 ROC curves for the four technique combinations. (a) LFR FTR 532R 532B, (b) LFR FTR 532R NIR, (c) LFR FTR 532B NIR, (d) LFR 532R 532B NIR and (e) FTR 532R 532B NIR. 
Table S 1 Summary of the PLSR model performance when correlating anti-tTG antibody levels to the spectroscopic data.

\begin{tabular}{|c|c|c|c|c|c|c|c|c|c|c|}
\hline \multirow{2}{*}{$\begin{array}{l}\text { Number } \\
\text { of } \\
\text { techniques } \\
\text { combined }\end{array}$} & \multirow[t]{2}{*}{ Technique } & \multirow{2}{*}{$\begin{array}{c}\text { \# of } \\
\text { factors }\end{array}$} & \multicolumn{4}{|c|}{ Calibration } & \multicolumn{4}{|c|}{ Cross-validation } \\
\hline & & & Slope & Offset & RMSE & $\begin{array}{c}\text { R- } \\
\text { square }\end{array}$ & Slope & Offset & RMSE & $\begin{array}{c}\text { R- } \\
\text { square }\end{array}$ \\
\hline \multirow{3}{*}{1} & FTR & 5 & 0.90 & 6.1 & 16 & 0.90 & 0.65 & 20 & 32 & 0.61 \\
\hline & $532 \mathrm{R}$ & 5 & 0.81 & 12 & 22 & 0.81 & 0.57 & 30 & 40 & 0.40 \\
\hline & 532B & 3 & 0.22 & 48 & 45 & 0.22 & 0.16 & 53 & 48 & 0.13 \\
\hline \multirow{6}{*}{2} & LFR 532R & 6 & 0.86 & 8.4 & 19 & 0.86 & 0.43 & 37 & 44 & 0.29 \\
\hline & LFR 532B & 3 & 0.41 & 36 & 39 & 0.41 & 0.22 & 49 & 48 & 0.14 \\
\hline & LFR NIR & 3 & 0.59 & 25 & 32 & 0.59 & 0.44 & 36 & 40 & 0.40 \\
\hline & FTR 532R & 6 & 0.87 & 5.1 & 18 & 0.87 & 0.64 & 24 & 34 & 0.56 \\
\hline & FTR 532B & 6 & 0.90 & 6.2 & 16 & 0.90 & 0.62 & 21 & 34 & 0.57 \\
\hline & FTR NIR & 3 & 0.62 & 24 & 31 & 0.62 & 0.53 & 30 & 37 & 0.49 \\
\hline \multirow[t]{10}{*}{3} & LFR FTR 532R & 6 & 0.87 & 8 & 18 & 0.87 & 0.56 & 28 & 36 & 0.49 \\
\hline & LFR FTR 532B & 5 & 0.78 & 13 & 24 & 0.78 & 0.50 & 32 & 39 & 0.44 \\
\hline & LFR FTR NIR & 5 & 0.81 & 12 & 22 & 0.81 & 0.62 & 24 & 34 & 0.56 \\
\hline & LFR 532R 532B & 6 & 0.87 & 8 & 18 & 0.87 & 0.48 & 34 & 42 & 0.34 \\
\hline & LFR 532R NIR & 3 & 0.63 & 23 & 31 & 0.63 & 0.48 & 32 & 39 & 0.42 \\
\hline & LFR 532B NIR & 3 & 0.63 & 23 & 31 & 0.63 & 0.50 & 32 & 38 & 0.47 \\
\hline & FTR 532R 532B & 6 & 0.87 & 7.9 & 18 & 0.87 & 0.67 & 21 & 34 & 0.55 \\
\hline & FTR 532R NIR & 3 & 0.65 & 21 & 30 & 0.65 & 0.54 & 29 & 37 & 0.51 \\
\hline & FTR 532B NIR & 3 & 0.62 & 23 & 31 & 0.62 & 0.52 & 30 & 36 & 0.50 \\
\hline & 532R 532B NIR & 3 & 0.61 & 24 & 31 & 0.61 & 0.50 & 31 & 38 & 0.44 \\
\hline \multirow[t]{2}{*}{4} & LFR FTR 532R 532B & 6 & 0.87 & 8.0 & 18 & 0.87 & 0.58 & 27 & 36 & 0.52 \\
\hline & LFR FTR 532R NIR & 5 & 0.80 & 12 & 23 & 0.80 & 0.61 & 24 & 34 & 0.55 \\
\hline
\end{tabular}


Table S 2 Recalculated diagnosis performance based on the 4 level histology SVM and grouping different severities together. $\mathrm{TP}=$ true positive, $\mathrm{TN}=$ true negative, $\mathrm{FP}=$ false positive and $\mathrm{FN}=$ false negative.

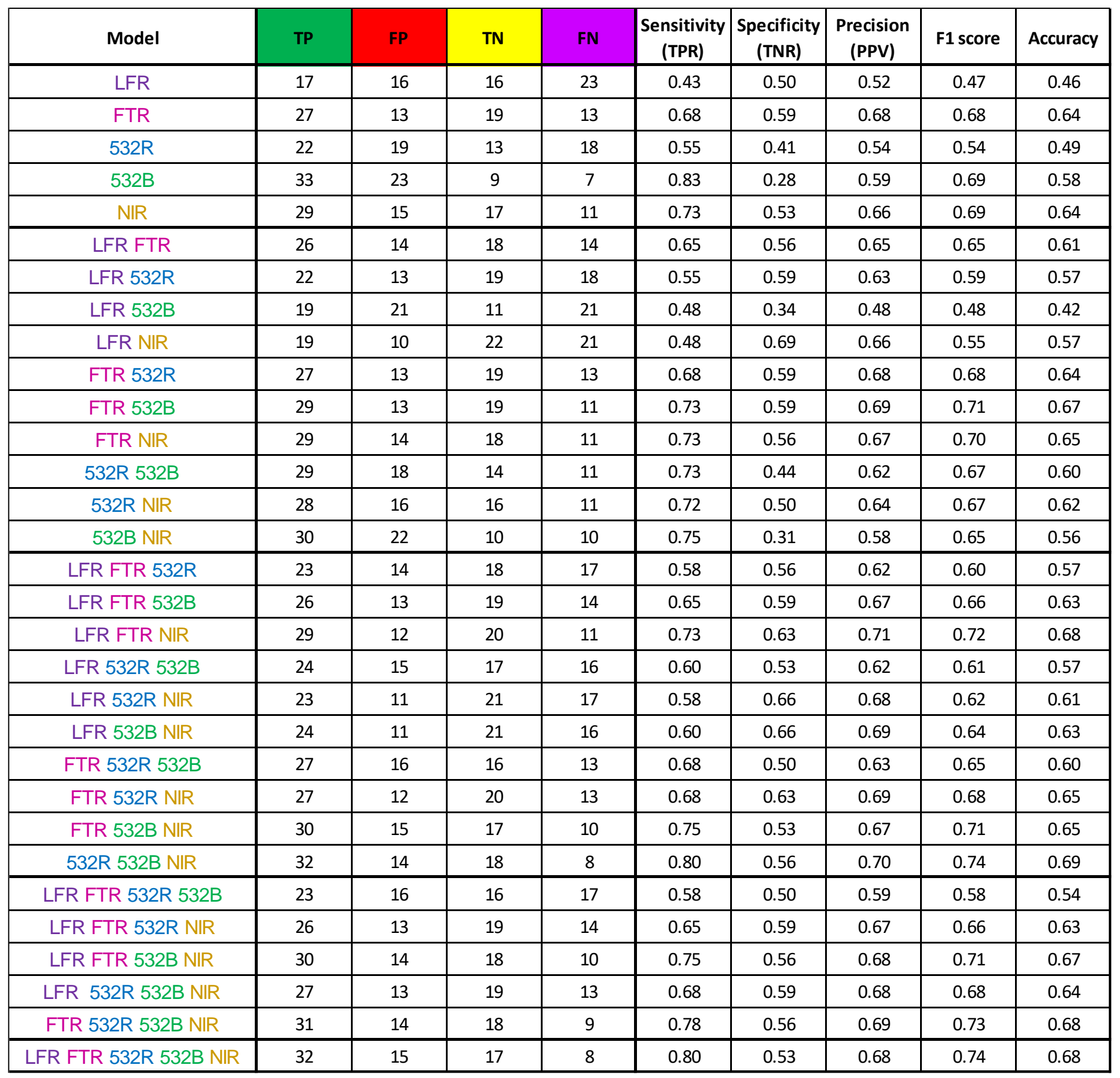


Table S 3 Diagnosis based SVM performances by participant (classification per participant is the majority from individual biopsies). $\mathrm{TP}=$ true positive, $\mathrm{TN}=$ true negative, $\mathrm{FP}=$ false positive and $\mathrm{FN}=$ false negative.

\begin{tabular}{|c|c|c|c|c|c|c|c|c|c|}
\hline $\begin{array}{c}\text { SVM } 2 \text { levels, fold by } \\
\text { participant, majority rules } \\
\text { classification }\end{array}$ & TP & FP & $\mathrm{TN}$ & FN & Accuracy & F1 score & $\begin{array}{c}\text { Precision } \\
\text { (PPV) }\end{array}$ & $\begin{array}{c}\text { Sensitivity } \\
\text { (TPR) }\end{array}$ & $\begin{array}{c}\text { Specificity } \\
\text { (TNR) }\end{array}$ \\
\hline LFR & 5 & 4 & 5 & 8 & 0.45 & 0.45 & 0.56 & 0.38 & 0.56 \\
\hline FTR & 10 & 3 & 6 & 3 & 0.73 & 0.77 & 0.77 & 0.77 & 0.67 \\
\hline $532 R$ & 4 & 5 & 5 & 9 & 0.39 & 0.36 & 0.44 & 0.31 & 0.50 \\
\hline 532B & 4 & 5 & 5 & 9 & 0.39 & 0.36 & 0.44 & 0.31 & 0.50 \\
\hline NIR & 10 & 4 & 6 & 3 & 0.70 & 0.74 & 0.71 & 0.77 & 0.60 \\
\hline LFR FTR & 10 & 5 & 4 & 3 & 0.64 & 0.71 & 0.67 & 0.77 & 0.44 \\
\hline LFR 532R & 5 & 6 & 4 & 6 & 0.43 & 0.45 & 0.45 & 0.45 & 0.40 \\
\hline LFR 532B & 8 & 6 & 4 & 4 & 0.55 & 0.62 & 0.57 & 0.67 & 0.40 \\
\hline LFR NIR & 6 & 4 & 6 & 6 & 0.55 & 0.55 & 0.60 & 0.50 & 0.60 \\
\hline FTR 532R & 8 & 3 & 7 & 4 & 0.68 & 0.70 & 0.73 & 0.67 & 0.70 \\
\hline FTR 532B & 8 & 4 & 5 & 5 & 0.59 & 0.64 & 0.67 & 0.62 & 0.56 \\
\hline FTR NIR & 10 & 4 & 5 & 3 & 0.68 & 0.74 & 0.71 & 0.77 & 0.56 \\
\hline 532R 532B & 7 & 4 & 6 & 5 & 0.59 & 0.61 & 0.64 & 0.58 & 0.60 \\
\hline 532R NIR & 11 & 6 & 5 & 2 & 0.67 & 0.73 & 0.65 & 0.85 & 0.45 \\
\hline 532B NIR & 7 & 5 & 4 & 5 & 0.52 & 0.58 & 0.58 & 0.58 & 0.44 \\
\hline LFR FTR 532R & 8 & 5 & 5 & 4 & 0.59 & 0.64 & 0.62 & 0.67 & 0.50 \\
\hline LFR FTR 532B & 9 & 4 & 5 & 4 & 0.64 & 0.69 & 0.69 & 0.69 & 0.56 \\
\hline LFR FTR NIR & 11 & 3 & 7 & 2 & 0.78 & 0.81 & 0.79 & 0.85 & 0.70 \\
\hline LFR 532R 532B & 8 & 4 & 5 & 3 & 0.65 & 0.70 & 0.67 & 0.73 & 0.56 \\
\hline LFR 532R NIR & 9 & 3 & 7 & 4 & 0.70 & 0.72 & 0.75 & 0.69 & 0.70 \\
\hline LFR 532B NIR & 8 & 3 & 6 & 5 & 0.64 & 0.67 & 0.73 & 0.62 & 0.67 \\
\hline FTR 532R 532B & 8 & 3 & 6 & 4 & 0.67 & 0.70 & 0.73 & 0.67 & 0.67 \\
\hline FTR 532R NIR & 10 & 2 & 7 & 3 & 0.77 & 0.80 & 0.83 & 0.77 & 0.78 \\
\hline FTR 532B NIR & 9 & 4 & 5 & 4 & 0.64 & 0.69 & 0.69 & 0.69 & 0.56 \\
\hline 532R 532B NIR & 11 & 3 & 7 & 2 & 0.78 & 0.81 & 0.79 & 0.85 & 0.70 \\
\hline LFR FTR 532R 532B & 9 & 4 & 5 & 3 & 0.67 & 0.72 & 0.69 & 0.75 & 0.56 \\
\hline LFR FTR 532R NIR & 11 & 3 & 6 & 2 & 0.77 & 0.81 & 0.79 & 0.85 & 0.67 \\
\hline LFR FTR 532B NIR & 10 & 3 & 7 & 3 & 0.74 & 0.77 & 0.77 & 0.77 & 0.70 \\
\hline LFR 532R 532B NIR & 10 & 3 & 7 & 3 & 0.74 & 0.77 & 0.77 & 0.77 & 0.70 \\
\hline FTR 532R 532B NIR & 10 & 3 & 6 & 2 & 0.76 & 0.80 & 0.77 & 0.83 & 0.67 \\
\hline LFR FTR 532R 532B NIR & 11 & 3 & 7 & 2 & 0.78 & 0.81 & 0.79 & 0.85 & 0.70 \\
\hline
\end{tabular}



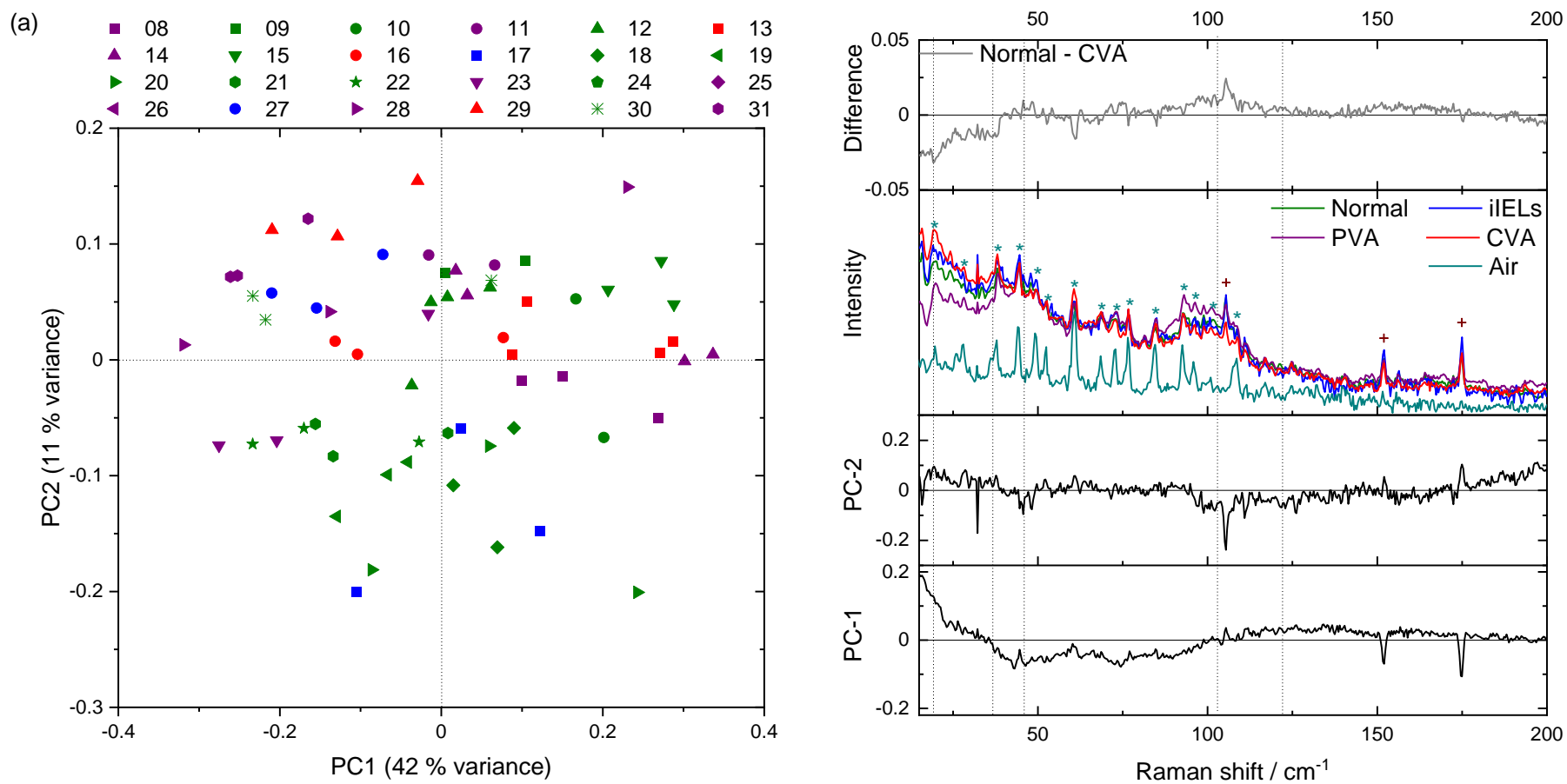

Figure S 1 PCA of the LFR spectroscopic dataset. (a) Scores plot of PC1 versus PC2 and (b) the associated loadings and comparative average spectrum per sample group. Please note the * indicates rotational spectra of air.

(a)

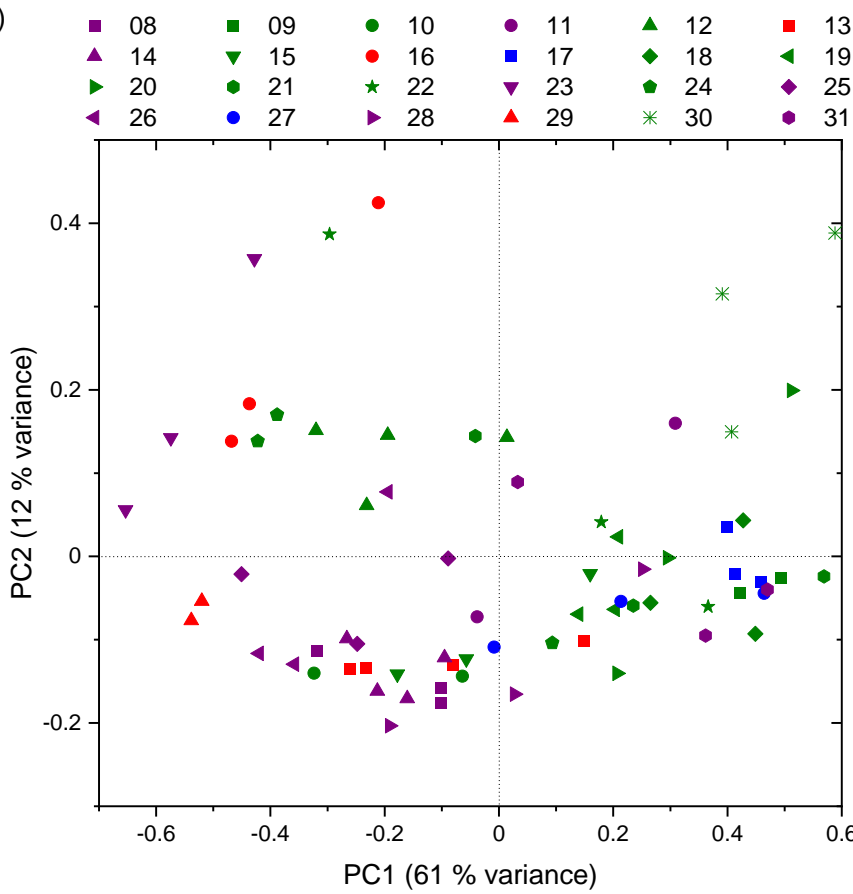

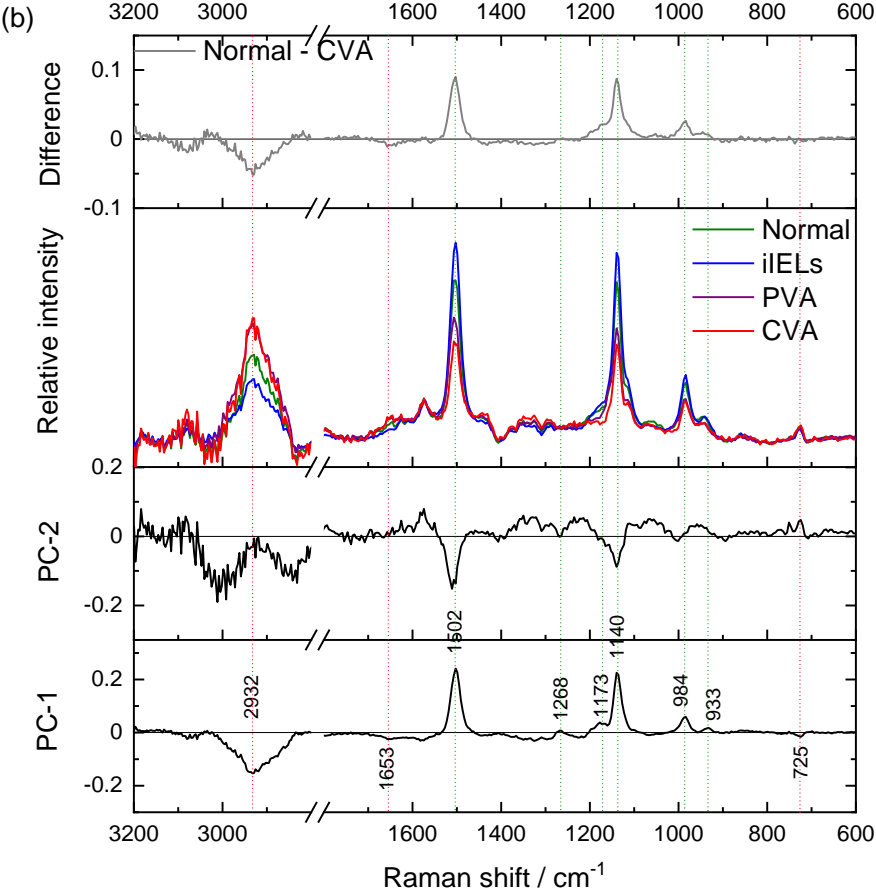

Figure S 2 PCA of the 532R spectroscopic dataset. (a) Scores plot of PC1 versus PC2 and (b) the associated loadings and comparative average spectrum per sample group. 

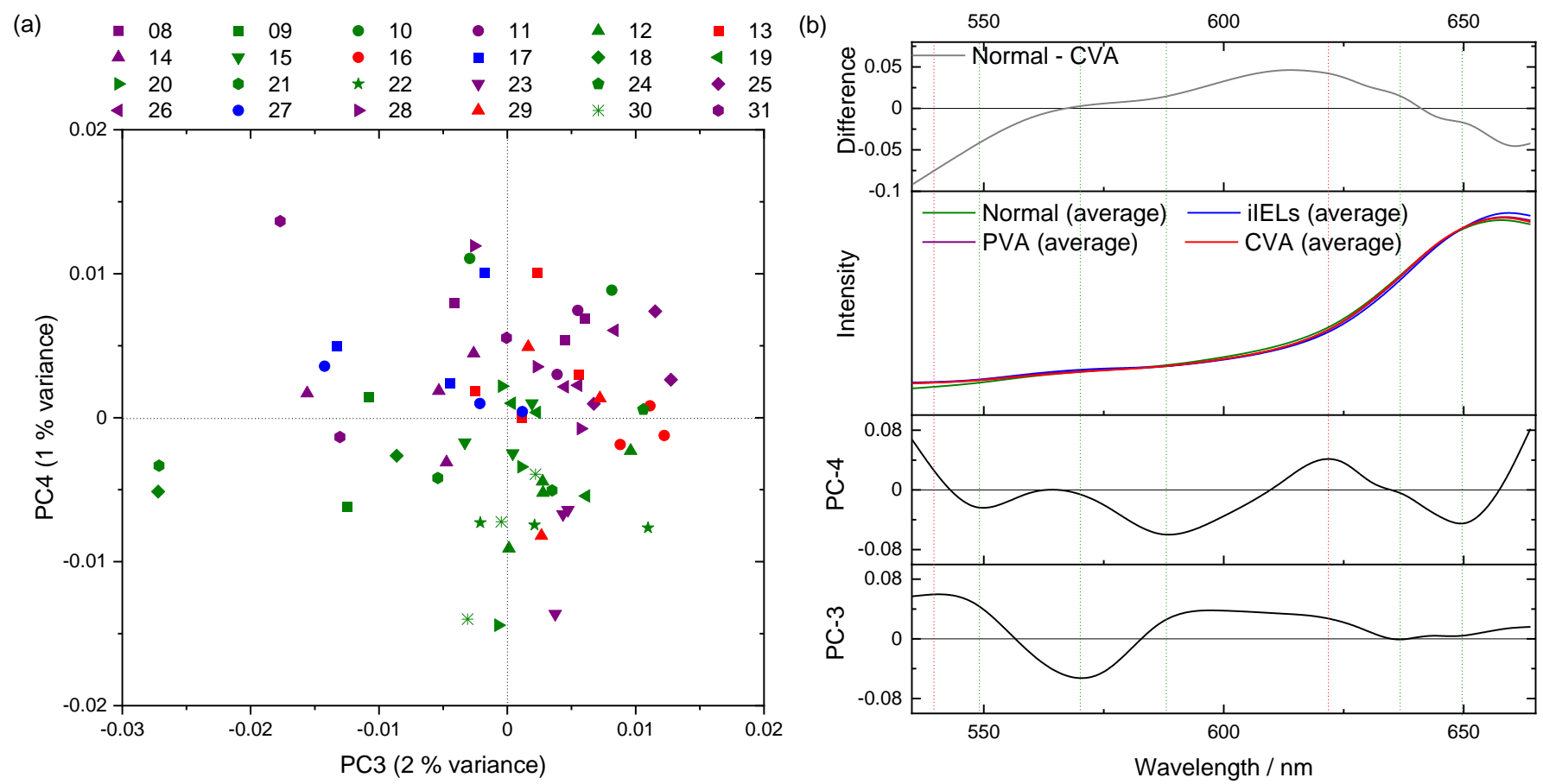

Figure S 3 PCA of the 532B spectroscopic dataset. (a) Scores plot of PC3 versus PC5 and (b) the associated loadings and comparative average spectrum per sample group.
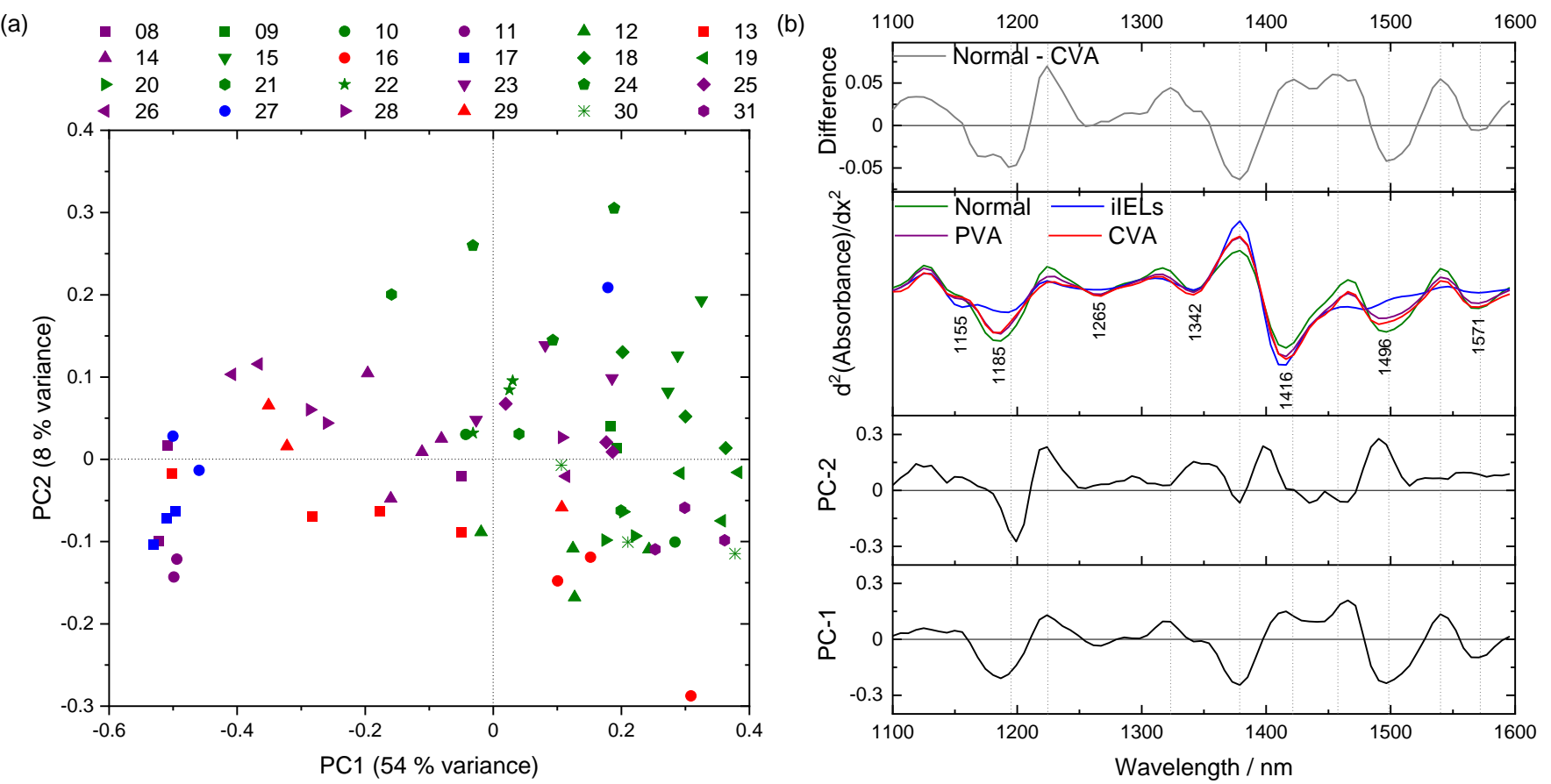

Figure S 4 PCA of the NIR spectroscopic dataset. (a) Scores plot of PC1 versus PC2 and (b) the associated loadings and comparative average spectrum per sample group. 

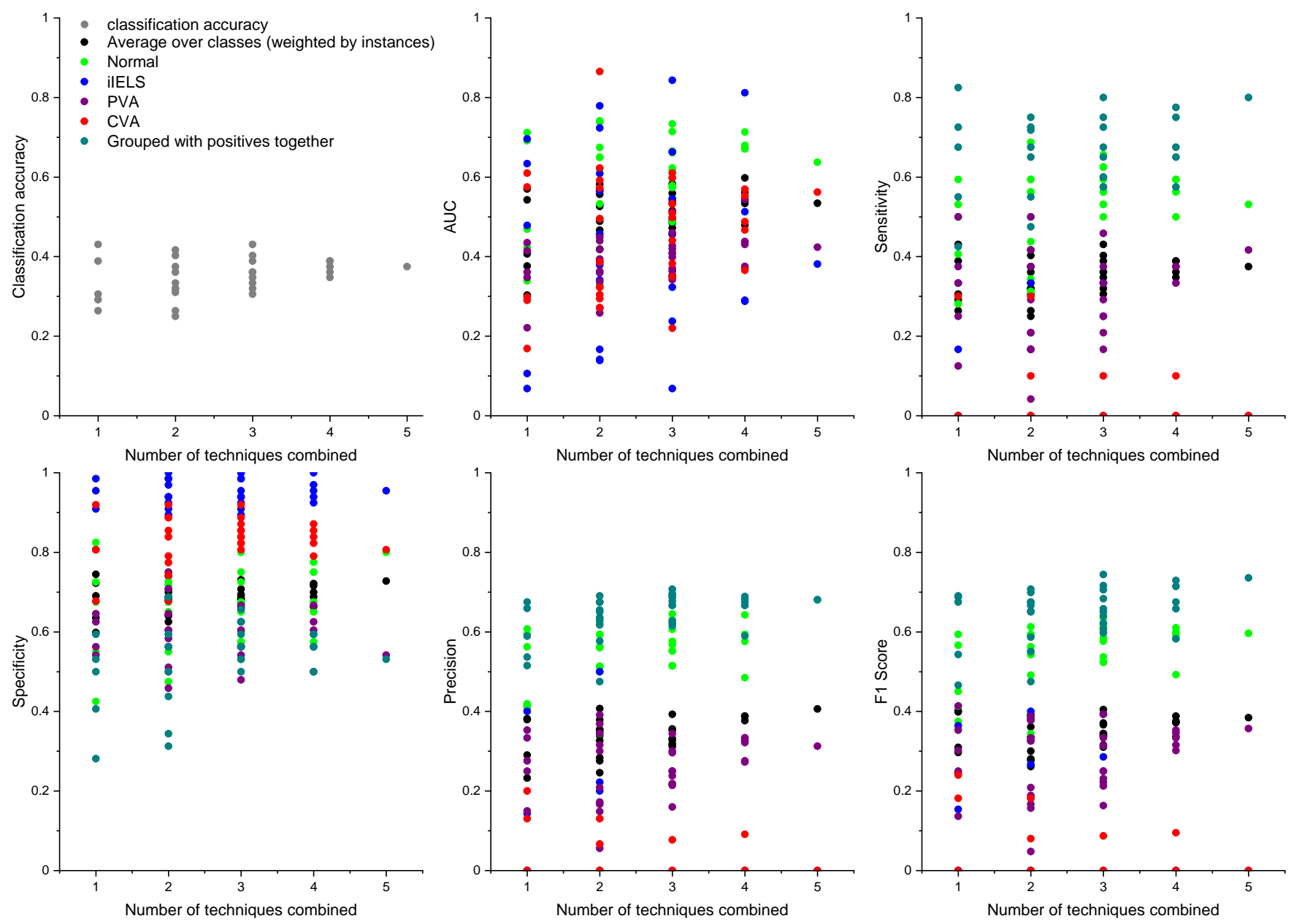

Figure S 5 Summary of the SVM-histology performance statistics versus number of techniques coupled.

\begin{tabular}{|c|c|c|c|c|c|}
\cline { 3 - 6 } \multicolumn{2}{|c|}{} & \multicolumn{4}{c|}{ Predicted } \\
\cline { 3 - 6 } & Normal & ilELS & PVA & CVA \\
\hline \multirow{3}{*}{ Actual } & Normal & 18 & 0 & 12 & 2 \\
\cline { 2 - 6 } & ilELS & 2 & 0 & 4 & 0 \\
\cline { 2 - 6 } & PVA & 9 & 0 & 12 & 3 \\
\cline { 2 - 6 } & CVA & 0 & 0 & 10 & 0 \\
\hline
\end{tabular}

\begin{tabular}{|c|c|}
\hline TP & 29 \\
\hline TN & 18 \\
\hline FP & 14 \\
\hline FN & 11 \\
\hline
\end{tabular}

Figure S 6 Example confusion matrix for the histology SVM and grouping different severities together. TP=true positive, TN $=$ true negative, $\mathrm{FP}=$ false positive and $\mathrm{FN}=$ false negative. 

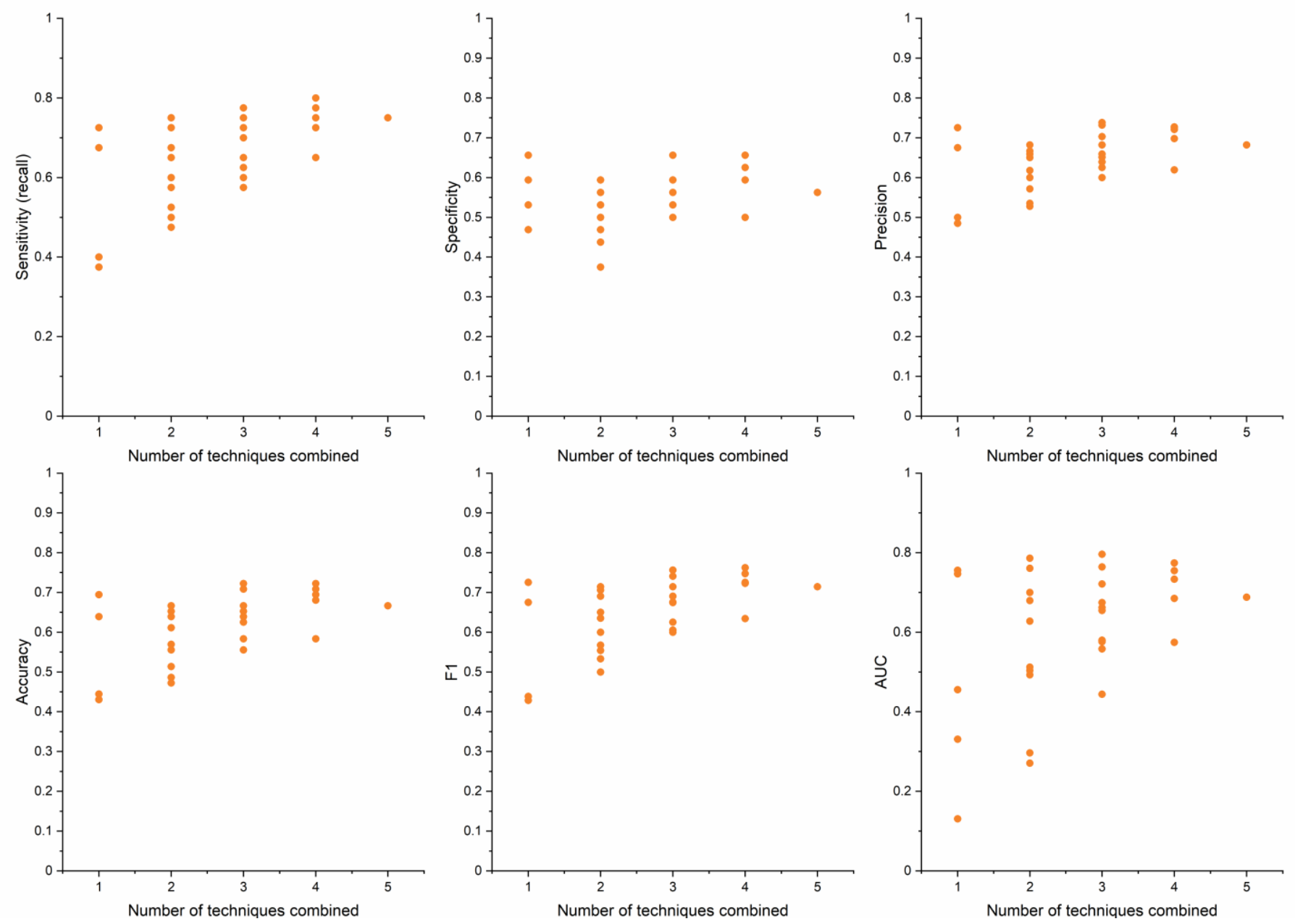

Figure S 7 Summary of the SVM-diagnosis performance statistics versus number of techniques coupled. 

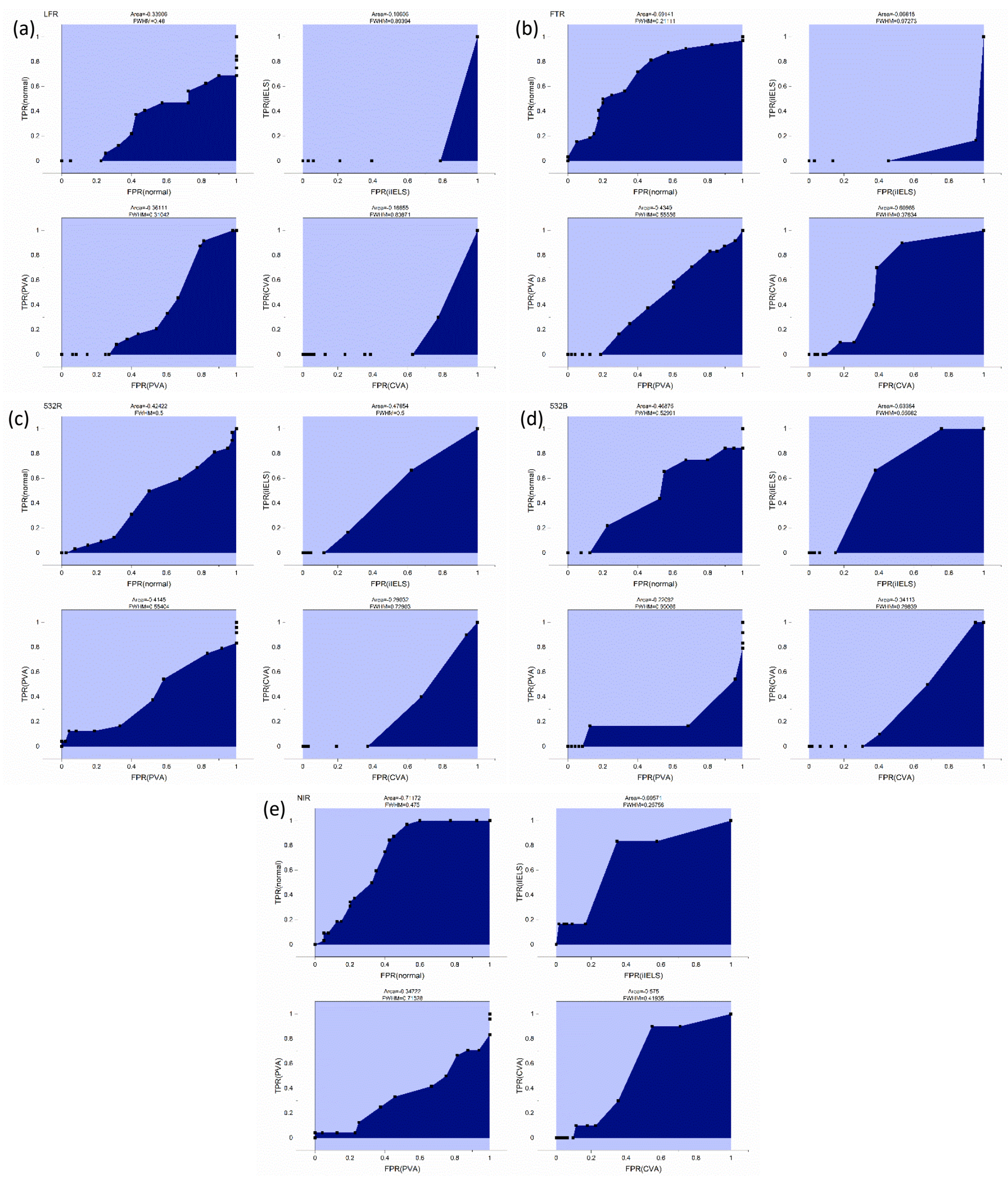

Figure S 8 ROC area under the curve for each class from SVM classification models with 4 histological levels - individual techniques. (a) LFR, (b) FTR, (c) 532R, (d) 532B and (e) NIR. 

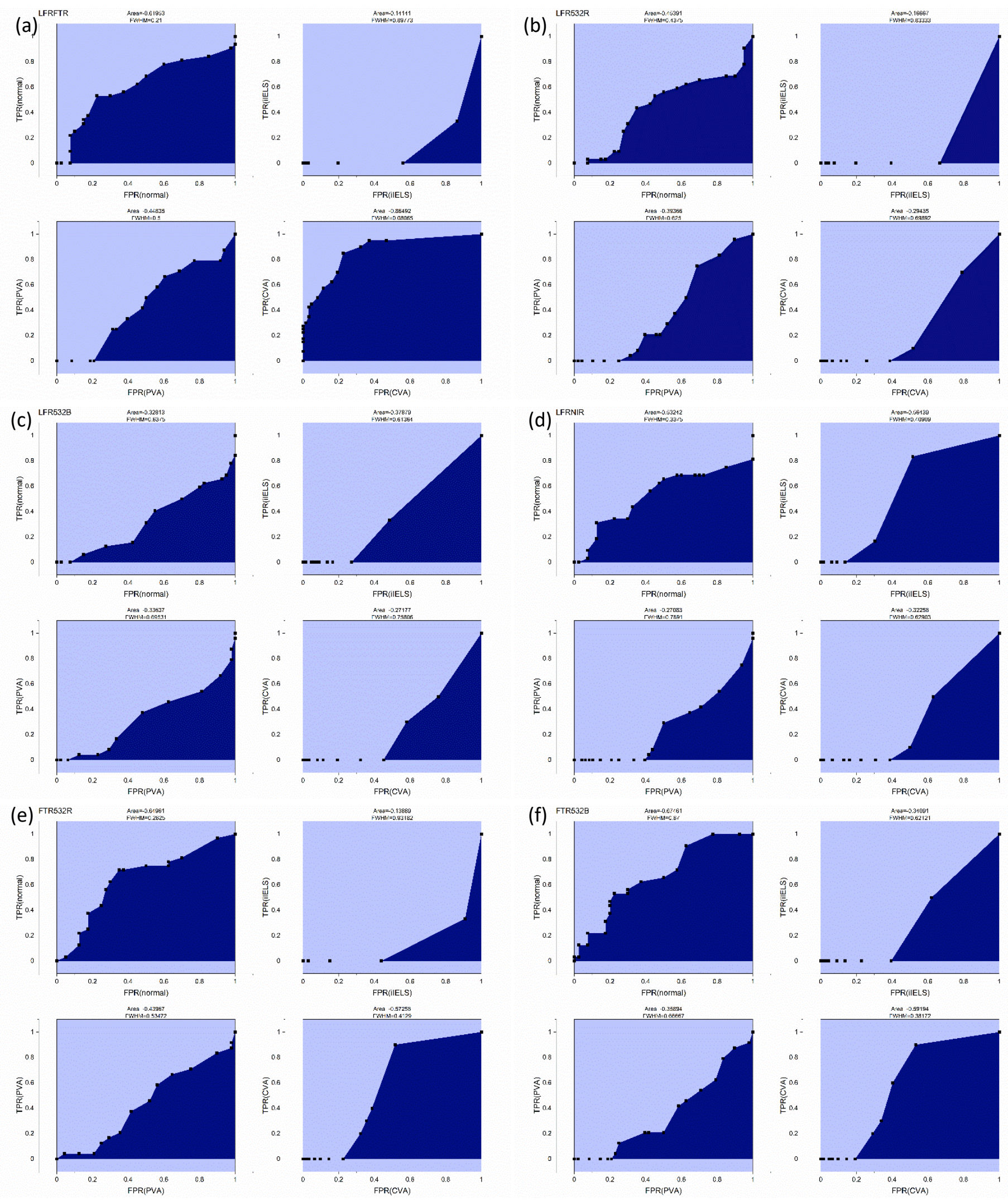

Supplementary information for Can Coupling Multiple Complementary Methods Improve the Spectroscopic Based Diagnosis of Gastrointestinal Illnesses? A Proof of Principle Ex Vivo Study Using Celiac Disease as the Model Illness. 

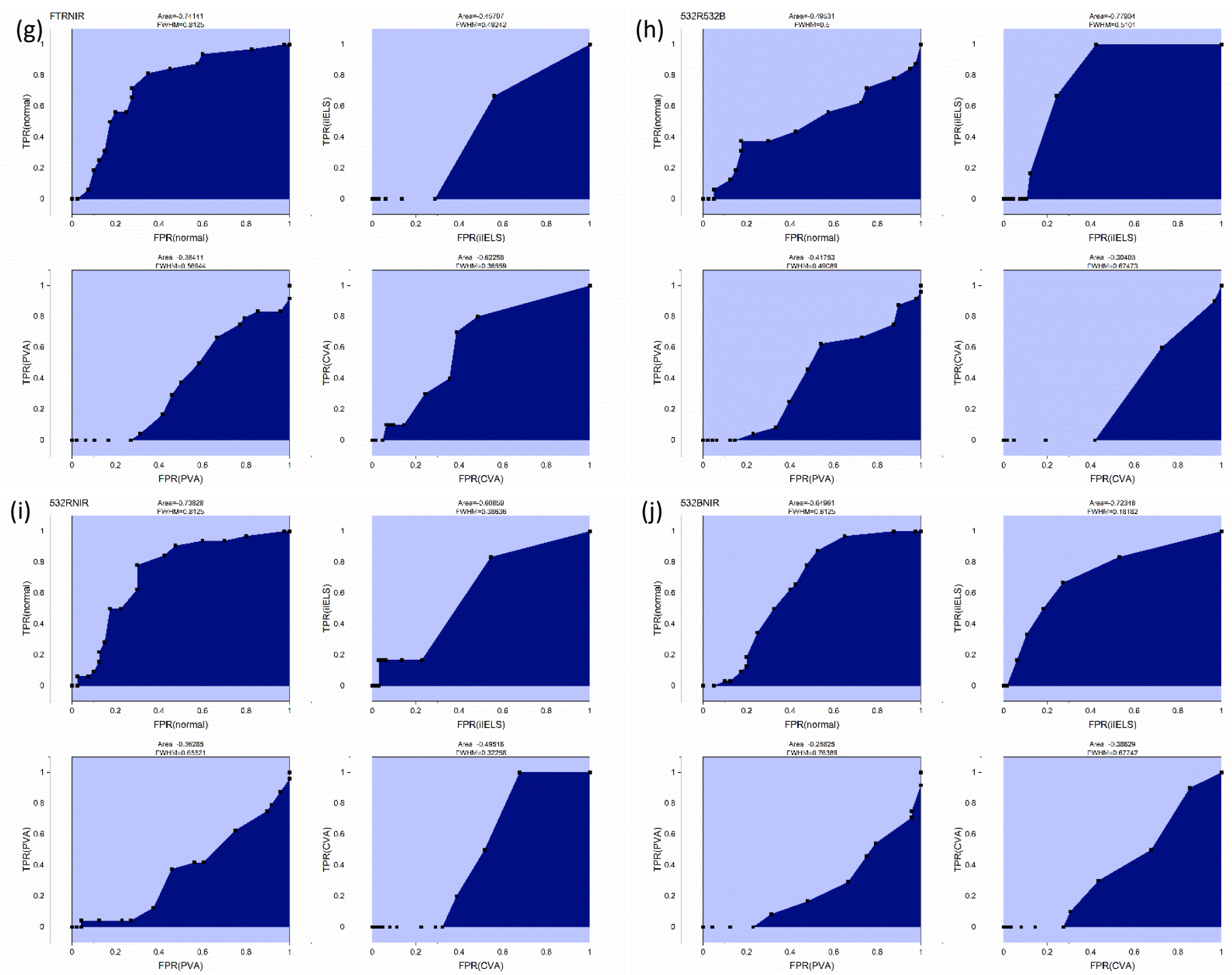

Figure S 9 ROC area under the curve for each class from SVM classification models with 4 histological levels - combining two techniques. (a) LFR FTR, (b) LFR 532R, (c) LFR 532B, (d) LFR NIR, (e) FTR 532R, (f) FTR 532B, (g) FTR NIR, (h) 532R 532B, (i) 532R NIR and (j) 532B NIR. 

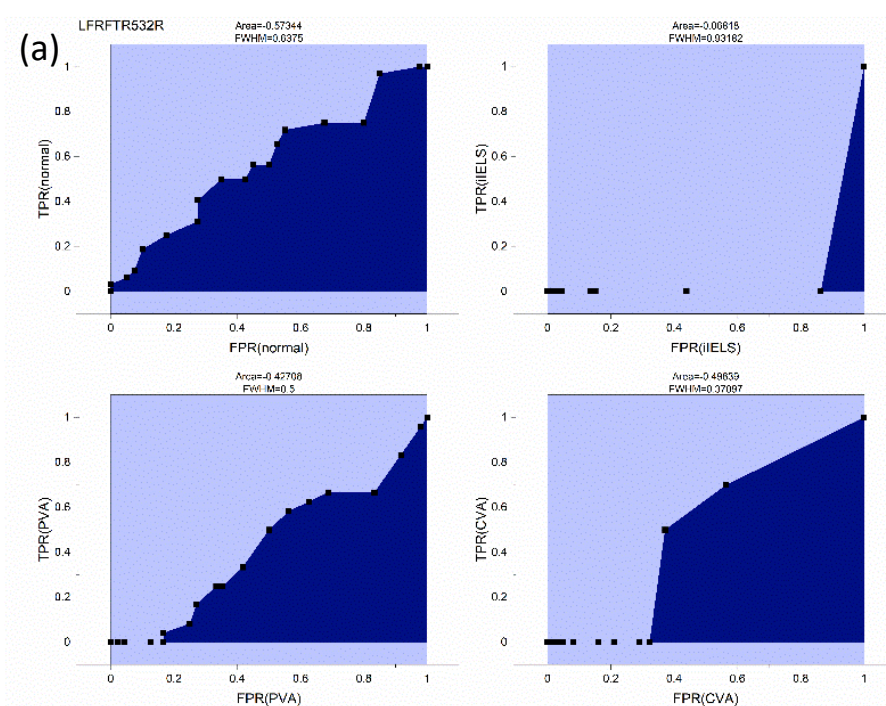

(c)
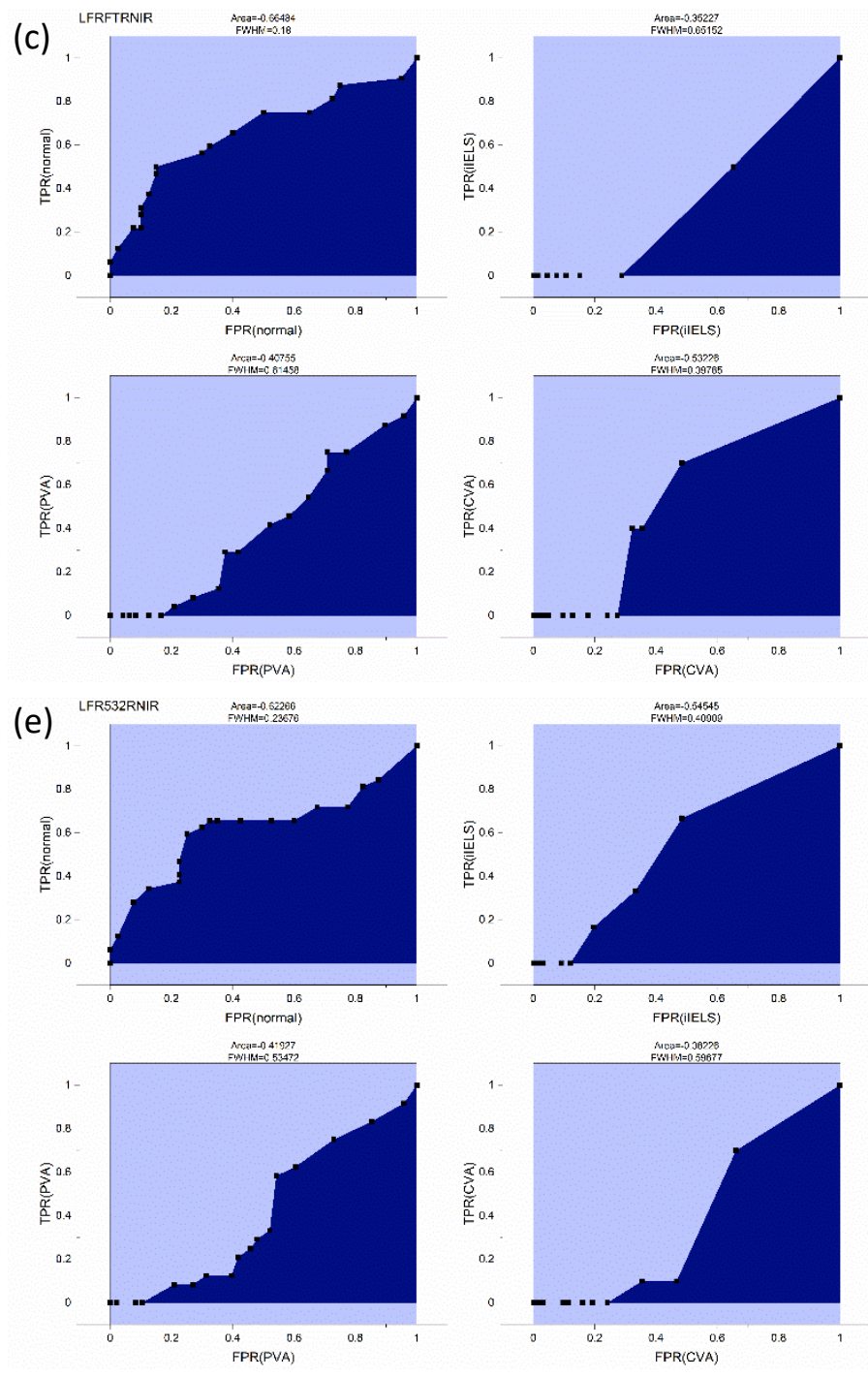
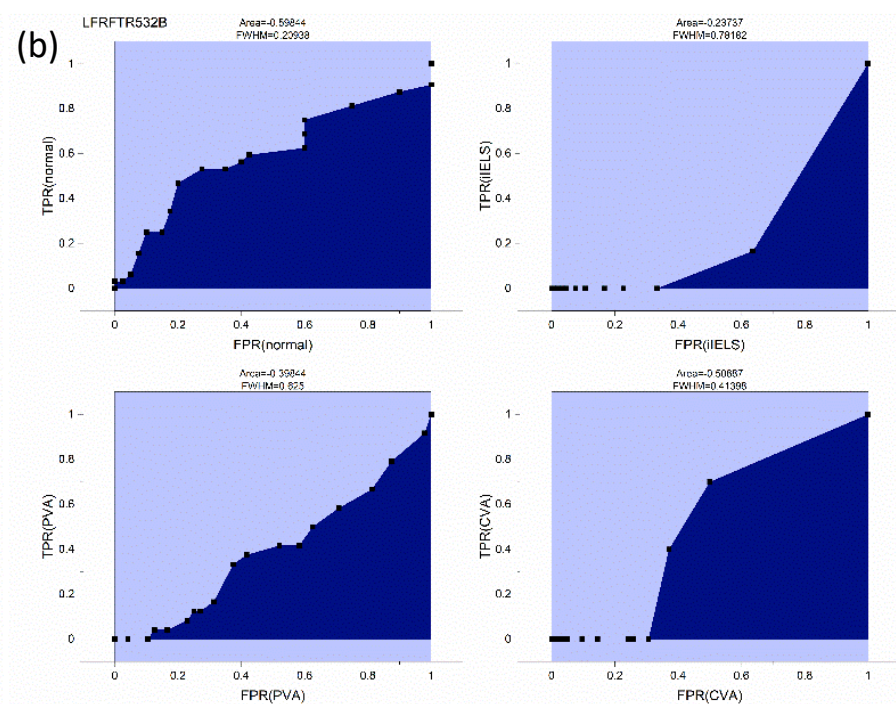

(d)
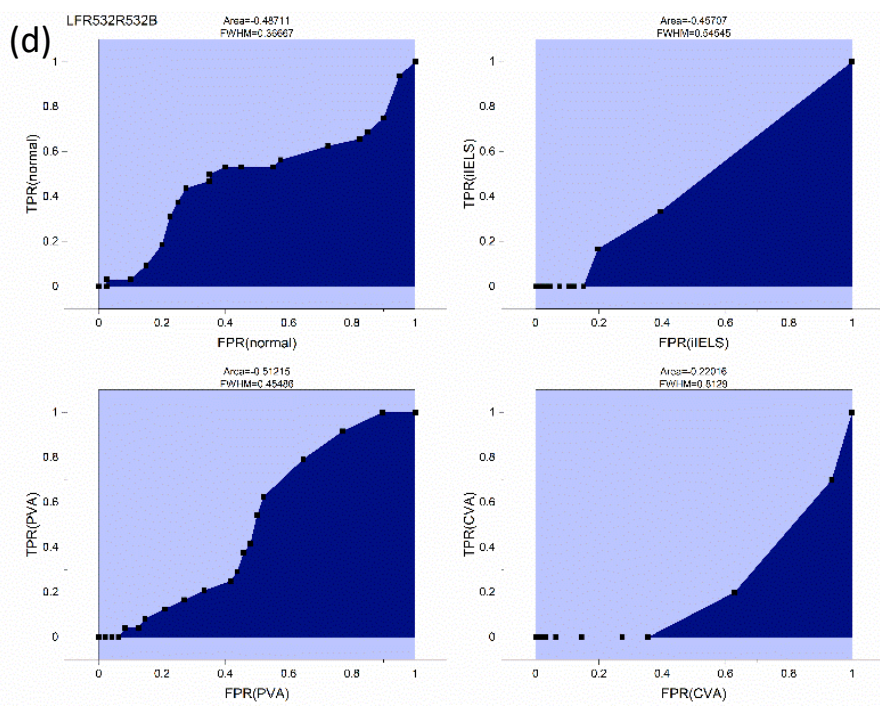

(f)
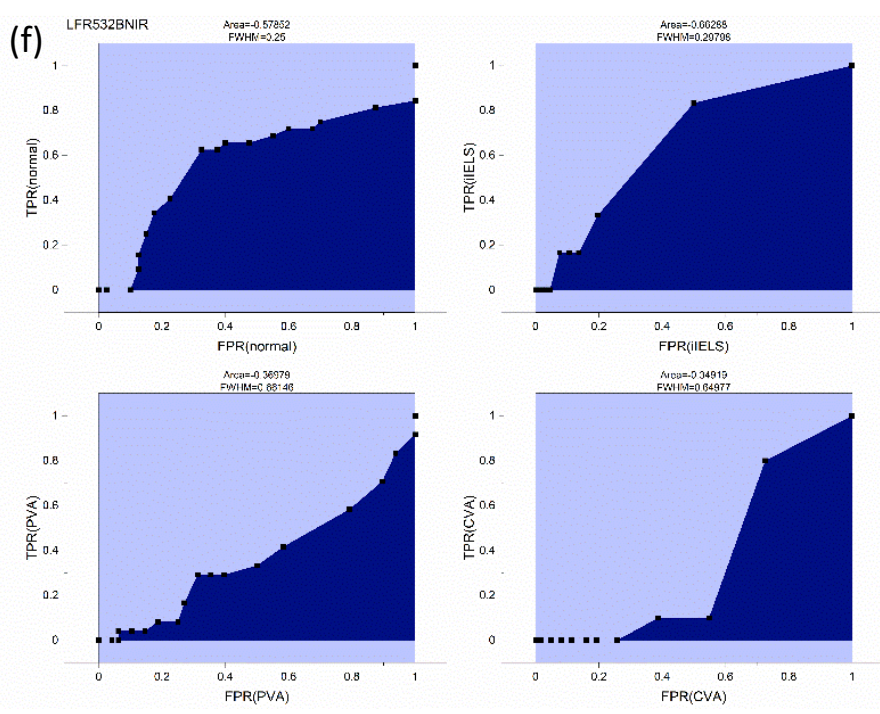

Supplementary information for Can Coupling Multiple Complementary Methods Improve the Spectroscopic Based Diagnosis of Gastrointestinal Illnesses? A Proof of Principle Ex Vivo Study Using Celiac Disease as the Model Illness. 

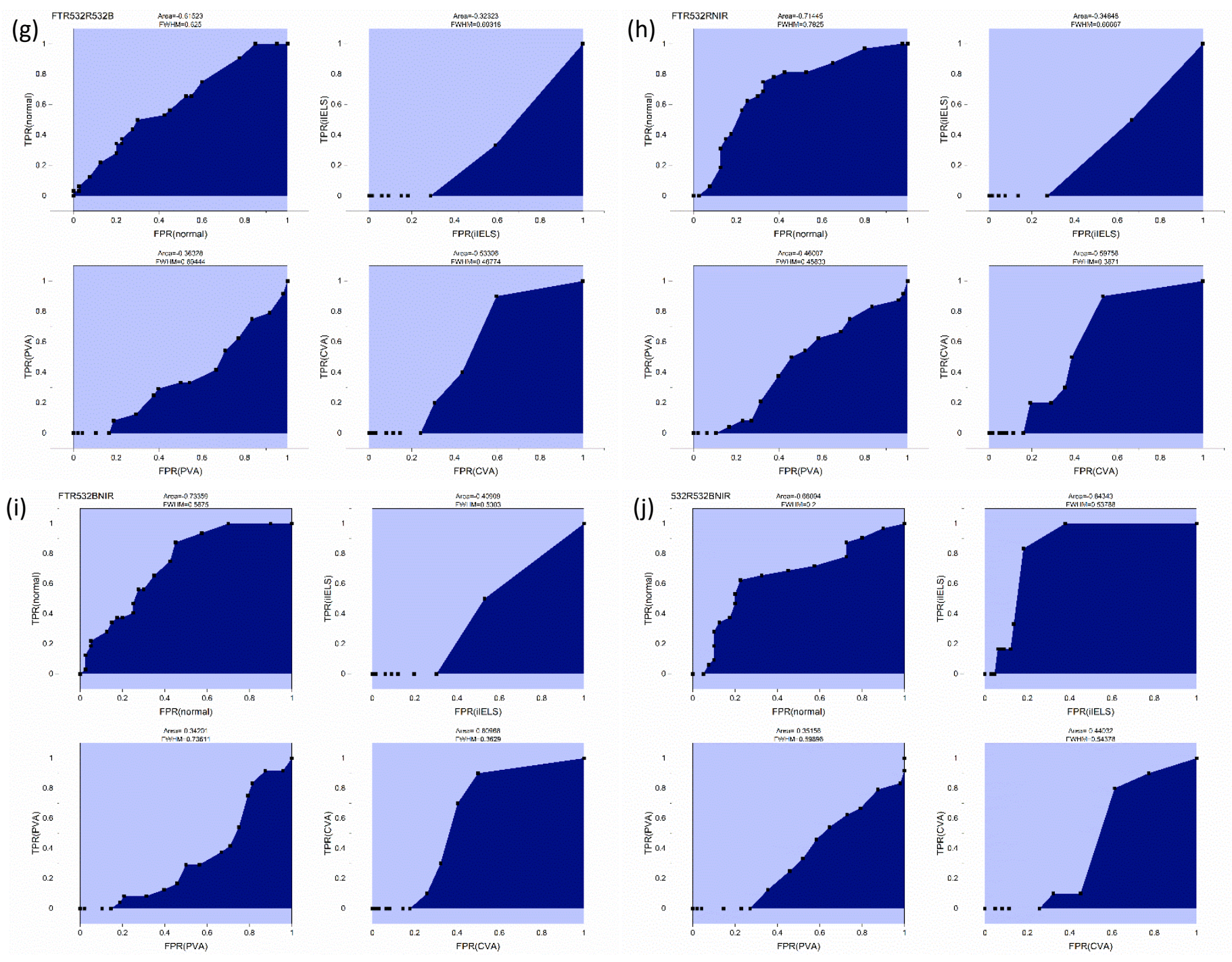

Figure S 10 ROC area under the curve for each class from SVM classification models with 4 histological levels - combining three techniques. (a) LFR FTR 532R, (b) LFR FTR 532B, (c) LFR FTR NIR, (d) LFR 532R 532B, (e) LFR 532R NIR, (f) LFR 532B NIR, (g) FTR 532R 532B, (h) FTR 532R NIR, (i) FTR 532B NIR, and (j) 532R 532B NIR. 

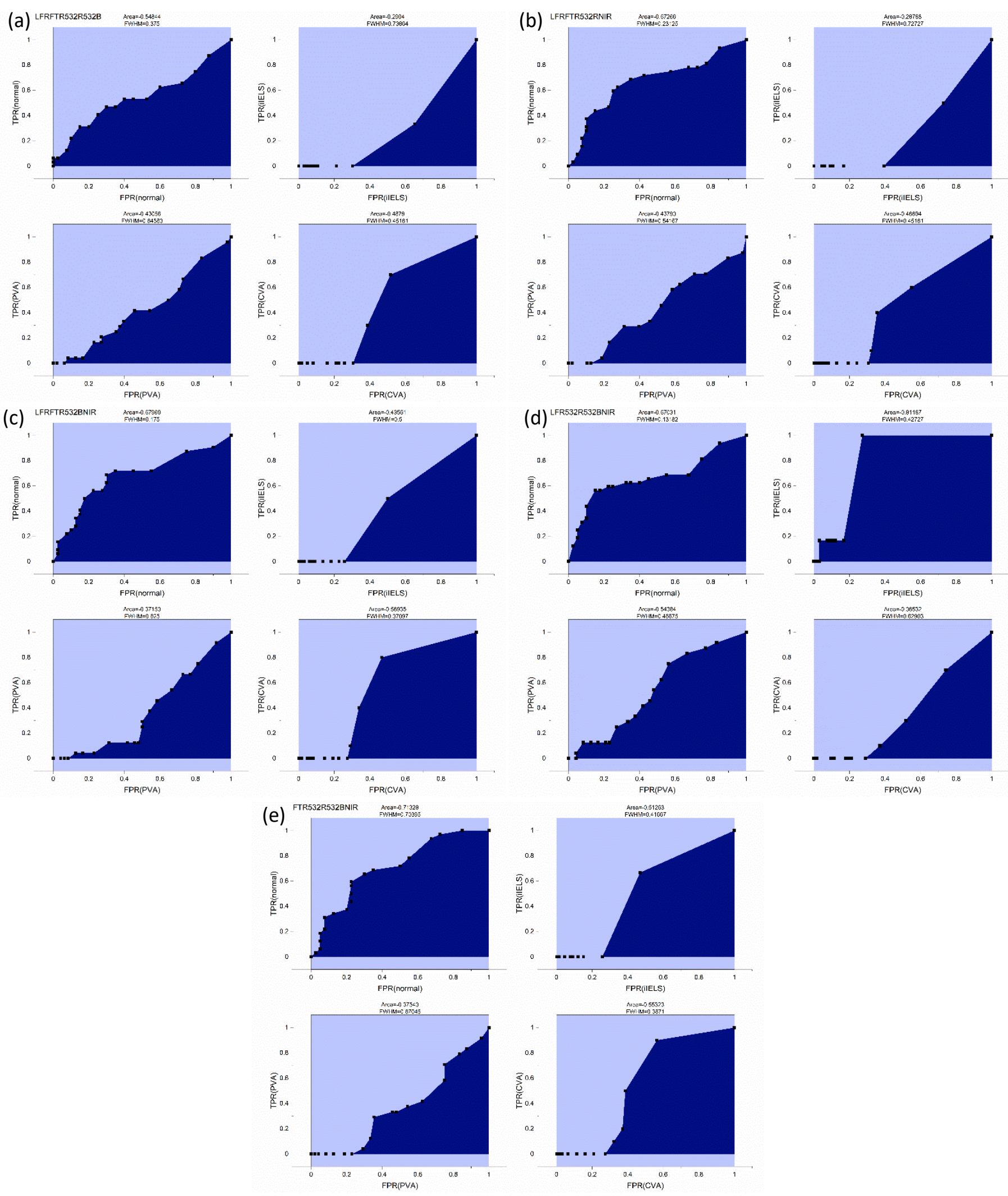

Figure S 11 ROC area under the curve for each class from SVM classification models with 4 histological levels - combining four techniques. (a) LFR FTR 532R 532B, (b) LFR FTR 532R NIR, (c) LFR FTR 532B NIR, (d) LFR 532R 532B NIR, and (e) FTR 532R 532B NIR. 

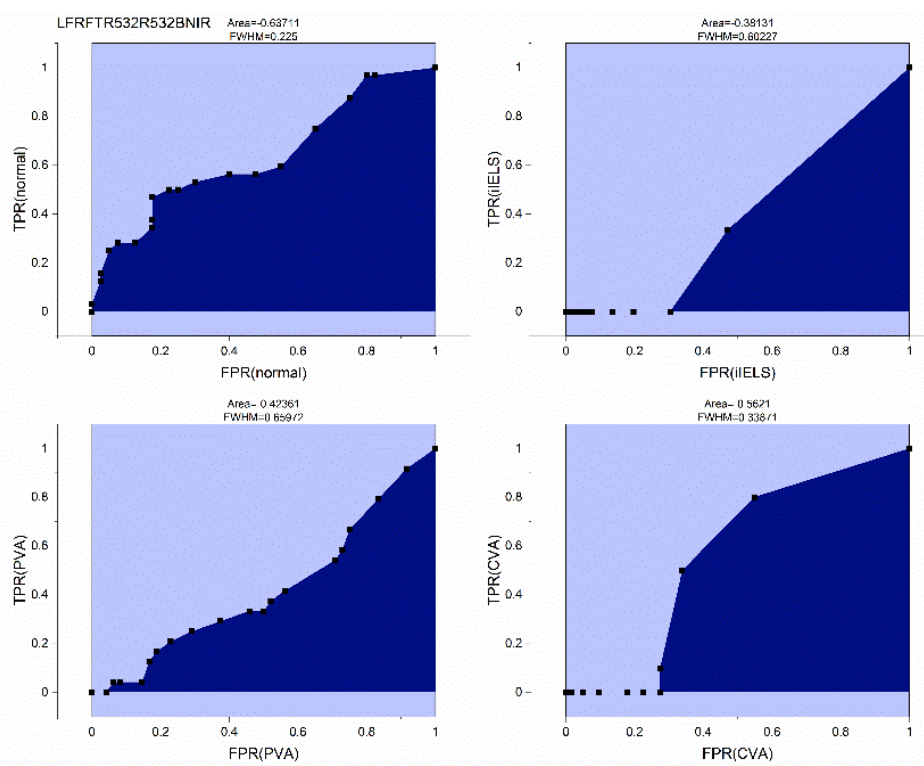

Figure S 12 ROC area under the curve for each class from SVM classification models with 4 histological levels - combining five techniques.
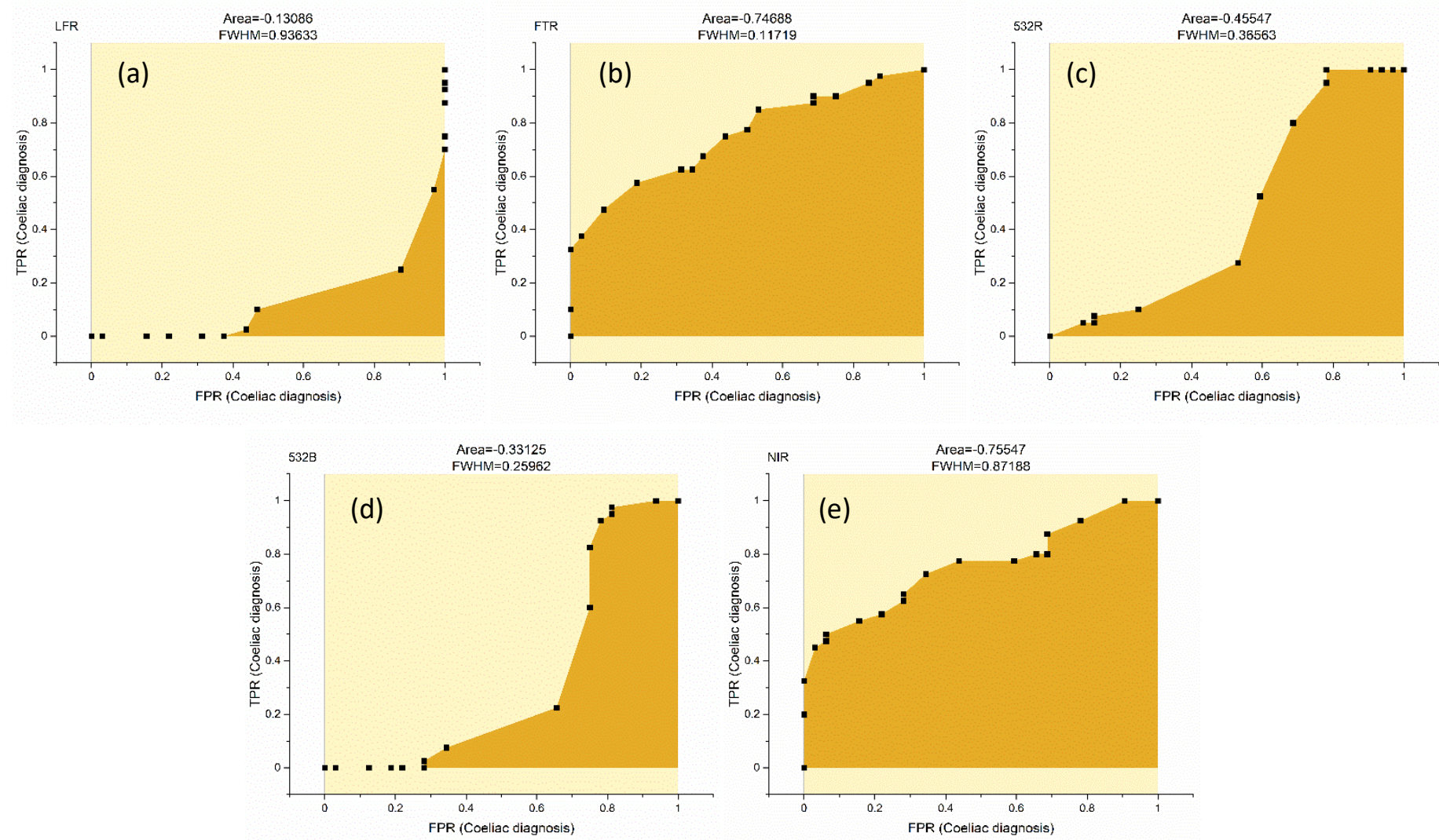

Figure S 13 ROC curves for the individual techniques. (a) LFR, (b) FTR, (c) 532R, (d) 532B, and (e) NIR. 

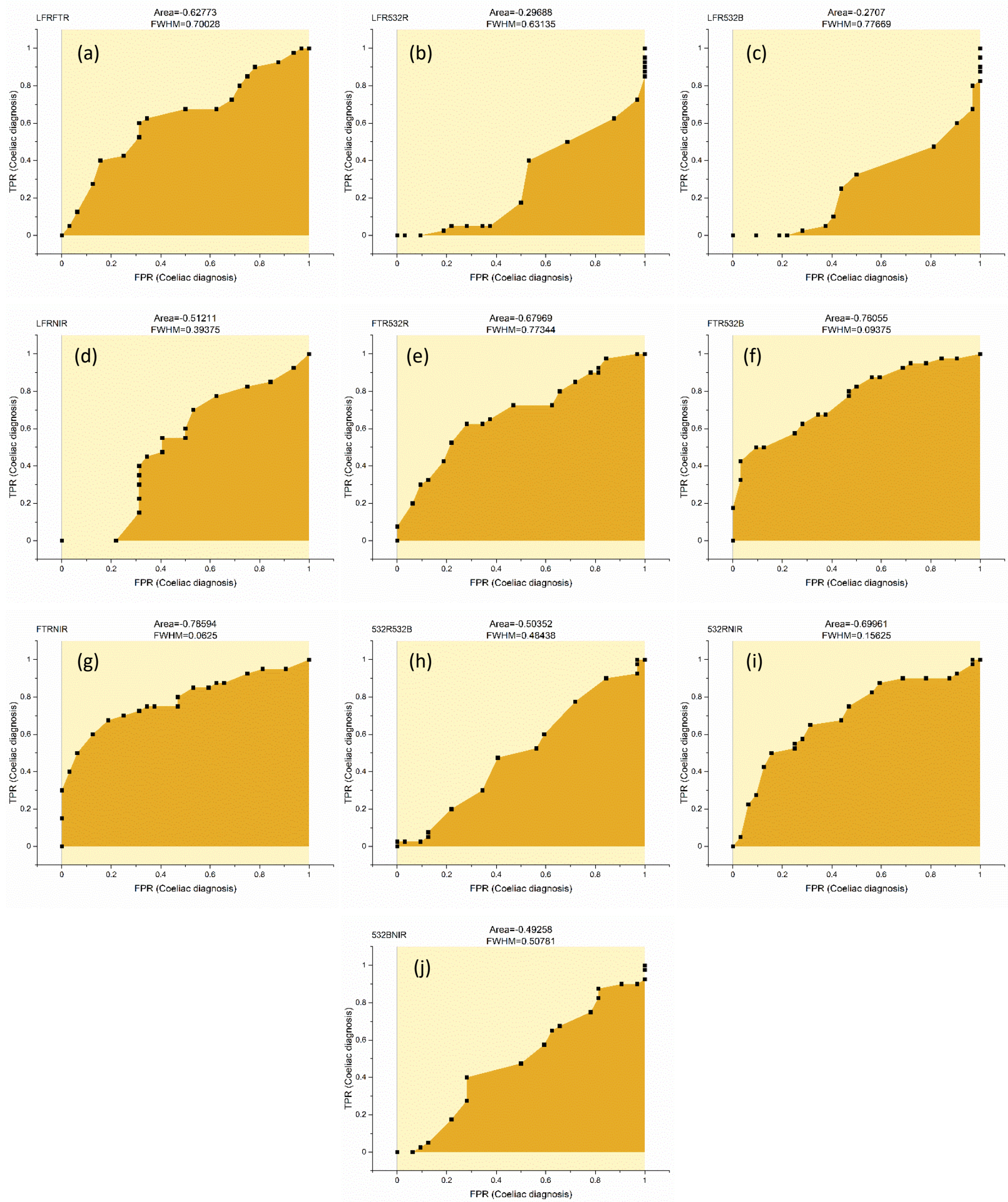

Figure S 14 ROC curves for the paired techniques. (a) LFR FTR, (b) LFR 532R, (c) LFR 532B, (d) LFR NIR, (e) FTR 532R, (f) FTR 532B, (g) FTR NIR, (h) 532R 532B, (i) 532R NIR and (j) 532B NIR. 

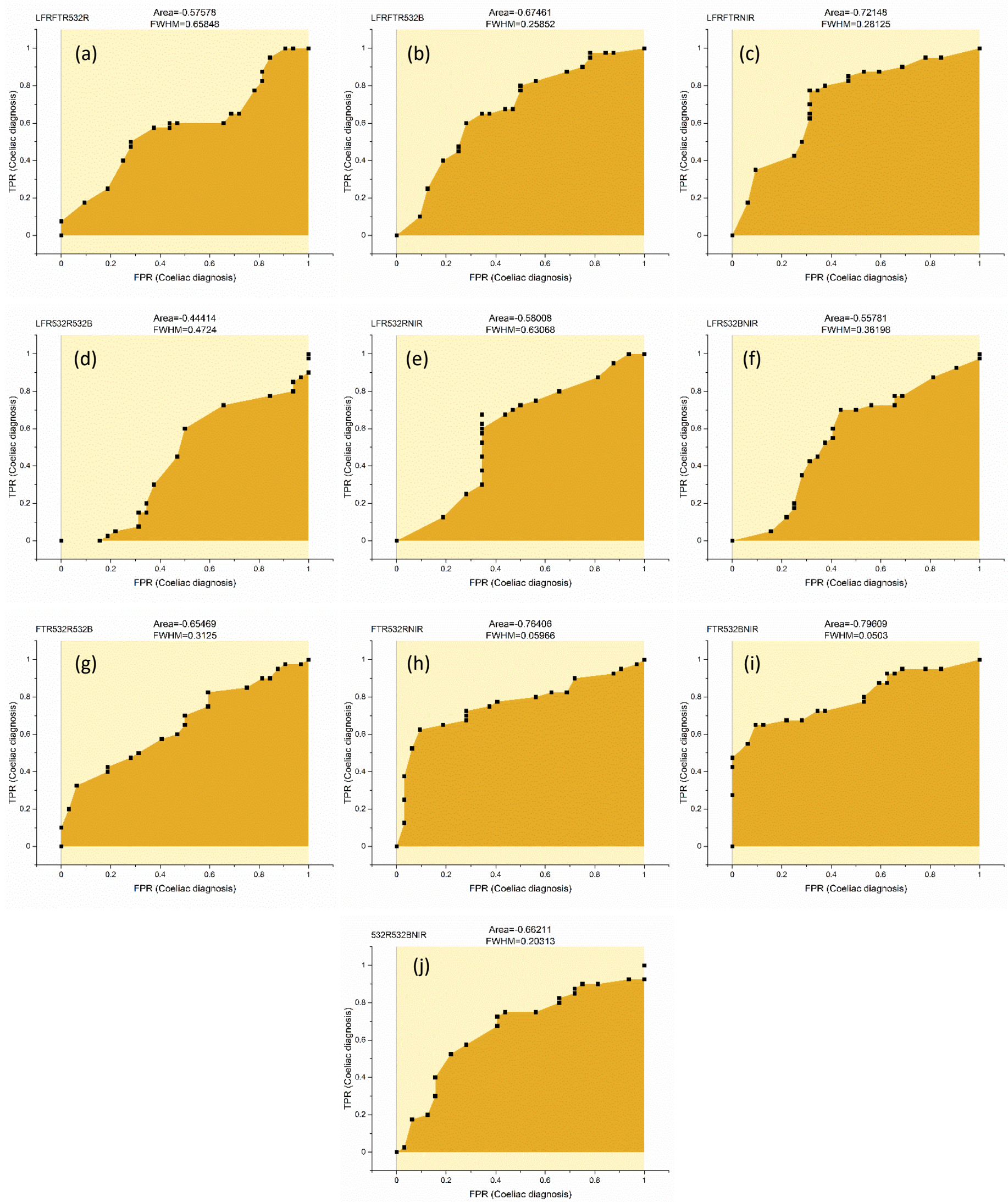

Figure S 15 ROC curves for the triple technique combinations. (a) LFR FTR 532R, (b) LFR FTR 532B, (c) LFR FTR NIR, (d) LFR 532R 532B, (e) LFR 532R NIR, (f) LFR 532B NIR, (g) FTR 532R 532B, (h) FTR 532R NIR, (i) FTR 532B NIR, and (j) 532R 532B NIR. 

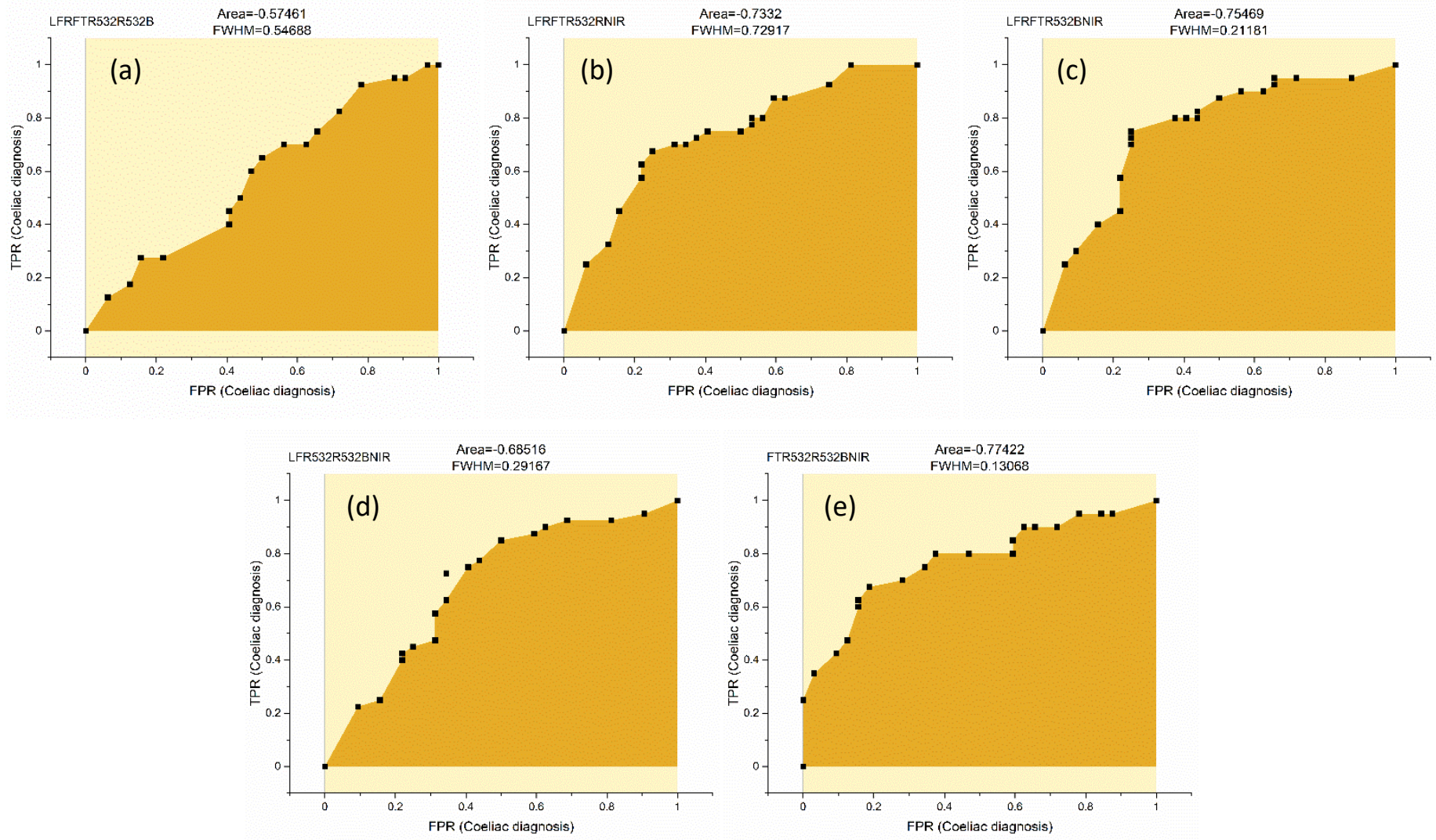

Figure $S 16$ ROC curves for the four technique combinations. (a) LFR FTR 532R 532B, (b) LFR FTR 532R NIR, (c) LFR FTR 532B NIR, (d) LFR 532R 532B NIR and (e) FTR 532R 532B NIR.

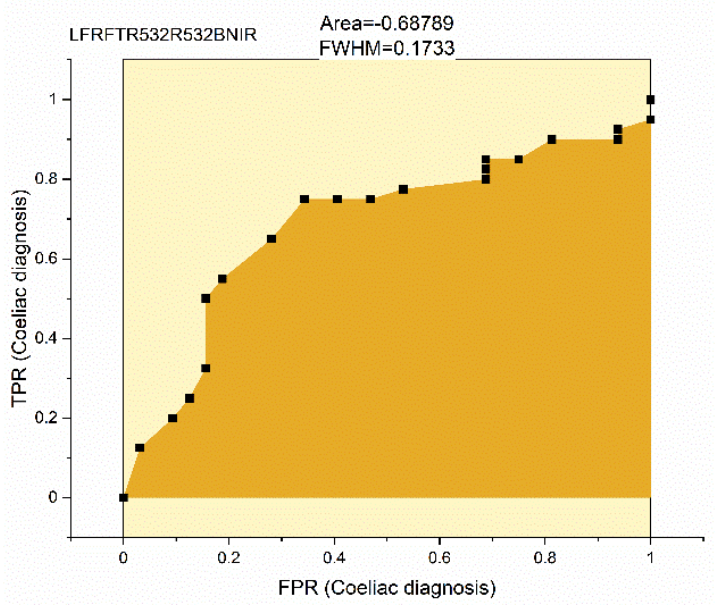

Figure S 17 ROC curve for the five technique combination (LFR FTR 532R 532B NIR). 